\title{
Mathematical challenges in integrative physiology
}

\author{
B. S. Brook - S. L. Waters
}

Published online: 9 February 2008

(C) Springer-Verlag 2008

\section{Introduction}

Traditional approaches to biomedical research are based on the subdivision of biological systems - by length (or time) scales (body, organ, tissue, cell, and molecule), discipline (biology, physiology, bioengineering, etc.), or sub-systems (cardiovascular, musculoskeletal, gastrointestinal, etc.). These subdivisions, however, make it difficult to unravel the systemic nature of the mechanisms that govern many of the (patho-) physiological processes in the human body. The challenge now is to complement this traditional approach with an integrative approach that makes it possible to combine observations, theories and predictions across dimensional scales, scientific disciplines, and anatomical sub-systems. This realisation has led to initiatives such as integrative biology [1], systems biology [2,3], the Physiome [4-6] and the Virtual Physiological Human [7]. These have highlighted the need to create a virtual infrastructure which should make it possible to interconnect predictive models defined at different scales, with different methods and with different levels of detail to enable investigation of systemic hypotheses.

Such a framework should also facilitate validation of theoretical or computational models by comparison with clinical or laboratory observations, by making the observation data easily accessible to the modelling community. Model validation is regularly overlooked and cannot be integrated fully into multi-scale models unless

B. S. Brook (凶)

School of Mathematical Sciences, University Park, University of Nottingham, Nottingham NG7 2RD, UK

e-mail: bindi.brook@nottingham.ac.uk

S. L. Waters

Oxford Centre for Industrial and Applied Mathematics, Mathematical Institute, 24-29 St Giles', Oxford, OX1 3LB, UK 
there is sufficient communication and exchange of data between the mathematical and life scientists.

\section{Multi-scale considerations}

Coupling predictive models throws up a multitude of mathematical challenges which are discussed in detail in the next section. To put them into context it is instructive to describe an exemplary case.

Asthma is characterised by airway hyper-responsiveness, chronic inflammation and remodelling. Airway narrowing, during an asthmatic attack, is caused by contraction of airway smooth muscle (ASM) cells lining the conducting airways. This narrowing response of the airways in asthmatic subjects to low doses of agonist (hyperresponsiveness) occurs over seconds. Over longer periods (months or years), repeated episodes of intense inflammation cause ASM proliferation leading to marked thickening of the airway wall (remodelling). The underlying biological mechanisms and genetic aspects responsible for hyper-responsiveness, inflammation and remodelling, however, continue to be debated. Thus new theoretical models that allow quantitative exploration of the roles and interactions of the genes, cells, tissues and chemical mediators involved in asthma are much needed. The processes involved occur across different spatial and temporal scales as follows.

Gene level: Various genes have been associated with an increased susceptibility to asthma. Environmental influences also play a role by interacting with the genetic predisposing factors. These genes will affect the make up of ASM cells thus affecting drug responsiveness, susceptibility to air pollutant, and airway remodelling through increased proliferation.

Molecular level: ASM contraction at the molecular level occurs as a result of the relative movement of actin and myosin filaments through forces generated by crossbridge attachment and detachment. The contractile response is triggered by intracellular calcium release.

Cellular level: In response to agonist stimulation a complex biochemical cascade occurs inside the cell (within seconds), resulting in the release of calcium from intracellular stores, which triggers the contractile response on a molecular level. Pharmacological interventions, such as application of bronchodilators, cause the relaxation of the airway smooth muscle through other intracellular signalling systems involving cyclic adenosine monophosphate (cAMP). Complex cross-talk occurs between these signalling pathways, which regulates ASM tone. Increased ASM proliferation governed by yet another signalling pathway leads to airway wall remodelling (over months) which in turn has an effect on macroscopic structural and flow properties.

Macroscopic level: Broncho-constriction during an asthma attack leads to airway obstruction and increased resistance to airflow. Clinical measures of hyperresponsiveness, e.g. dose-response curves and forced expiratory volume, are indicators of severity of asthma. These are linked to all of the above processes, e.g. the make-up of airway wall tissue and responsiveness is determined by genetic aspects which in turn affect molecular and cellular behaviour. Tissue biomechanics, fluid mechanics 
in the branching network of the lung and flow-structure interactions between the flow and airway wall are all important aspects of macroscopic pulmonary models for asthma.

\section{Future mathematical challenges}

In the example given above, the signalling pathways connecting problems at the gene level to the overall loss of lung function presents a formidable challenge in integrative multi-scale modelling. Similar examples of systemic processes that span temporal and spatial scales abound within the human body (e.g. from the generation of cardiac rhythm, to the pumping of blood by the heart and through to remodelling of the ventricles in heart-disease).

Multi-scale models require the use of a wide range of theoretical and numerical tools (cellular automata, agent-based modelling, stochastic processes, continuum mechanics, compartmental models, multiphase flows, computational fluid and solid dynamics). Furthermore, biological understanding is advancing at such a pace that provided the modelling responds appropriately to its complexity, this area of modelling will also lead to significant new mathematics.

(I) Multiscale behaviour. Effects from gene transcription (in the above example affecting susceptibility of airway smooth muscle to hyper-responsive behaviour) to organ-scale behaviour need to be addressed, with coupling in both directions and being mediated by an intermediate (i.e. cell) scale. For example gene-level effects determine the make up of the cells and tissue via the associated differentiation pathways while macro-scale distributions of stress or diffusible chemicals provide key determinants of individual cell behaviour. Sub-models developed at the relevant scale must interact with models developed at higher or lower scales. The challenge is thus:

(i) to interface different modelling frameworks at the same scale, building hybrid methods that combine and exploit the advantages brought by the different frameworks. For example, intracellular calcium signalling requires the combination of both stochastic processes and partial differential equation (PDE) methods;

(ii) to build nested models that can be used to transfer information between the different spatial scales. For example, cross-bridge models of force generation at the molecular level must be linked to airway contraction at the macroscopic level.

(II) Complex spatio-temporal networks. Signal-transduction pathways can be extremely involved and crosstalk (e.g. between the calcium and cAMP signalling pathway above) compounds their complexity and nonlinearity, as well as enhancing the advantages of mathematical approaches over purely intuitive ones. Models developed to understand genes require a wide range of modelling techniques that are already well-established (coupled ODEs) as well as the further development of complementary ones (Boolean networks, Petri nets, Bayesian networks, etc.). Such models are required, for example in asthma, to investigate the interaction of different candidate genes with environmental factors.

(III) Discreteness and stochasticity. Many important phenomena may be associated with, say, only a small number of molecules of a particular transcription factor being 
present in a cell and the standard partial differential equation (PDE) models may be inapplicable. Here stochastic models that account for stochastic events and small numbers are thus required. The robustness of model predictions to random perturbations will also need to be explored (for example in models of calcium signalling).

\section{Conclusion}

There is a clear need to bridge the gap between the traditional compartmentalised approach and the new integrative approach. This presents exciting new challenges for mathematicians working at the interface with engineering, biology and medicine. The demand here will be for entirely new models of sufficient realism that the biology is adequately captured, but not of such complexity that the results are inaccessible to intuition and interpretation. The crucial components of integrative multi-scale modelling are thus frameworks that are dictated by the biology and iterative model refinement through extensive experimental validation. Indeed such an integrative approach has already shed light on a key clinical problem; how heart arrhythmias that look very similar when recorded on an electrocardiogram can have many possible underlying causes [8].

\section{References}

1. Gavaghan, D.J., Simpson, A.C., Lloyd, S., MacRandal, D.F., Boyd, D.R.S.: Towards a grid infrastructure to support integrative approaches to biological research. Phil. Trans. R. Soc. A 363, 1829-1841 (2005)

2. Butcher, E.C., Berg, E.L., Kunkel, E.J.: Systems biology in drug discovery. Nature Biotech. 2, 12531259 (2004)

3. Ideker, T., Galitski, T., Hood, L.: A new approach to decoding life: systems biology. Annu. Rev. Genomics Hum. Genet. 2, 343-372 (2001)

4. Hunter, P., Nielsen, P.: A strategy for integrative computational physiology. Physiology 20, 316-325 (2005)

5. Hunter, P., Robbins, P., Noble, D.: The IUPS human physiome project. Eur. J. Physiol. 445, 9 (2002)

6. Hunter, P., Smith, N., Fernandez, J., Tawhai, M.: Integration from proteins to organs: the IUPS Physiome Project. Mech. Ageing Dev. 126, 187-192 (2005)

7. The STEP consortium. Seeding the Europhysiome: A Roadmap to the Virtual Physiological Human. On-line 5th July 2007. http://www.europhysiome.org/roadmap

8. The Heart Physiome Project. http://www.physiome.org.nz/heart 